\title{
An algorithm to determine the Heegaard genus of simple 3-manifolds with nonempty boundary
}

\author{
MARC LACKENBY
}

\begin{abstract}
We provide an algorithm to determine the Heegaard genus of simple 3-manifolds with nonempty boundary. More generally, we supply an algorithm to determine (up to ambient isotopy) all the Heegaard splittings of any given genus for the manifold. As a consequence, the tunnel number of a hyperbolic link is algorithmically computable. Our techniques rely on Rubinstein's work on almost normal surfaces, and also on a new structure called a partially flat angled ideal triangulation.
\end{abstract}

$57 \mathrm{~N} 10 ; 57 \mathrm{M} 25$

\section{Introduction}

The Heegaard genus of a compact orientable 3-manifold is an important invariant. The aim of this paper is to demonstrate that it is algorithmically computable, at least when the 3-manifold is simple and has nonempty boundary. Recall that a compact orientable 3-manifold is simple if it is irreducible and any properly embedded disc, incompressible annulus or incompressible torus is boundary parallel.

Theorem 1.1 Let $M$ be a compact connected orientable simple 3-manifold with nonempty boundary. Then there is an algorithm to determine the Heegaard genus of $M$. Moreover, for any given positive integer $n$, there is an algorithm to find all Heegaard surfaces for $M$ with genus at most $n$ (up to ambient isotopy).

This theorem can be applied to determine the tunnel number of hyperbolic links. Recall that a tunnel system for a link $L$ in $S^{3}$ is a collection of disjoint embedded arcs $t$ with $t \cap L=\partial t$, such that the exterior of $L \cup t$ is a handlebody. The tunnel number of $L$ is the minimal number of arcs in a tunnel system. Two tunnel systems $t_{1}$ and $t_{2}$ for $L$ are slide-equivalent if there is an isotopy of $S^{3}$ keeping $L$ fixed throughout, taking $N\left(L \cup t_{1}\right)$ to $N\left(L \cup t_{2}\right)$. The following corollary to Theorem 1.1 is proved in Section 5. 
Corollary 1.2 Let $L$ be a hyperbolic link in the 3-sphere. Then there is an algorithm to determine the tunnel number of $L$. Moreover, for any given positive integer $n$, there is an algorithm to find all tunnel systems for $L$ with at most $n$ arcs (up to slide-equivalence).

The input to the algorithms in Theorem 1.1 is a triangulation of $M$. In Corollary 1.2 , one may supply a diagram of the link or a triangulation of its exterior. Thus, the hyperbolic structure does not need to be given in advance. The second algorithm provided by Theorem 1.1 creates a finite list of Heegaard surfaces for $M$. More specifically, it provides an explicit subdivision of the triangulation of $M$ and explicit subcomplexes which are the required Heegaard surfaces. Note, however, there is no guarantee that the surfaces in this list are pairwise nonisotopic. This is because there is currently no known algorithm for determining whether two Heegaard surfaces for a 3-manifold are ambient isotopic.

Most of the key ideas behind this paper are due to Rubinstein. He proved that, given any triangulation of a compact orientable 3-manifold, any strongly irreducible Heegaard surface may be ambient isotoped into almost normal form [12]. Using the computable nature of normal surface theory, he explained how one might use this to compute the Heegaard genus of the manifold. However, the possible presence of normal tori creates formidable technical obstacles to this approach. Jaco and Rubinstein [3] have developed a theory of "1-efficient" and "layered" triangulations to try to overcome these difficulties, but this appears to be highly technical, and the results are not fully published. An alternative approach to Heegaard surfaces has been developed by Li [8; 7], starting with almost normal surfaces, but then using branched surfaces. Using this theory, he has solved some important longstanding problems. One of his theorems is as follows.

Theorem 1.3 [8] Any closed orientable irreducible atoroidal 3-manifold has only finitely many Heegaard splittings of a given genus, up to ambient isotopy.

However, his proof is nonconstructive, and so there appears to be no immediate way of finding all these Heegaard surfaces using his techniques. Our methods provide a proof of this result, but where the 3-manifold is compact, connected, orientable and simple and has nonempty boundary.

The algorithms given in this paper follow Rubinstein's original outline in many respects. Like Jaco and Rubinstein's approach, the key is to use triangulations with very restricted normal tori. But unlike their theory of 1-efficiency, the technique here is to use angle structures. We introduce "partially flat angled ideal triangulations", which have the key property that they contain no normal tori other than those that are normally parallel to 
a boundary component. We will show that any compact connected orientable simple 3-manifold with nonempty boundary (other than a 3-ball) has one of these ideal triangulations and that there is an algorithm to construct it.

In a paper such as this, it is particularly important to be clear about which parts are new and which are due to other mathematicians. The material in Section 2, where partially flat angled ideal triangulations are introduced, is new. However, similar notions have been used by other authors for other purposes (see for example Petronio and Weeks [11]). Theorem 2.2, which asserts that any compact connected orientable simple 3-manifold with nonempty boundary (other than a 3-ball) has a partially flat angled ideal triangulation and that this may be algorithmically constructed, is new. Section 3 contains mostly expository material relating to generalised Heegaard splittings. However, there are a number of important facts in this section which appear in print for the first time. These include Proposition 3.1, which states that, when one amalgamates a generalised Heegaard splitting, the resulting Heegaard splitting is independent of the choices that have been made. Additionally, we show that if the generalised Heegaard splitting is given, say, as a subcomplex of a triangulation of the 3-manifold, then the resulting Heegaard splitting is algorithmically constructible. In Section 4, we state that a generalised Heegaard splitting can be placed in normal and almost normal form, provided its even surfaces are incompressible and have no 2-sphere components and its odd surfaces are strongly irreducible. This is a mild generalisation of a well-known result of Rubinstein [12] and Stocking [16], and has essentially the same proof. We then describe the computational aspects of normal and almost normal surfaces in partially flat angled ideal triangulations. This is largely routine. In the final section, we draw these many threads together and describe the algorithms of Theorem 1.1 and Corollary 1.2.

Acknowledgements The author would like to thank the referee for some helpful suggestions which improved this paper. The author was supported by an EPSRC Advanced Research Fellowship.

\section{Partially flat angled ideal triangulations}

Angled ideal triangulations were first studied by Casson (unpublished), and then developed by the author in [6]. They are just an ideal triangulation, with an assignment of a real number to each edge of each ideal tetrahedron, satisfying some simple conditions. A mild generalisation of this concept, which we call a partially flat angled ideal triangulation, is a key ingredient of this paper.

An ideal tetrahedron is a tetrahedron with its vertices removed. An ideal triangulation of a 3-manifold $M$ is an expression of the interior of $M$ as a union of ideal tetrahedra 
with their faces glued homeomorphically in pairs. An angled ideal triangulation is an ideal triangulation, with a real number in the range $(0, \pi)$ assigned to each edge of each ideal tetrahedron, known as the interior angle of the edge, satisfying the following conditions:

(i) the angles at each ideal vertex of each ideal tetrahedron sum to $\pi$;

(ii) the angles around each edge sum to $2 \pi$.

In partially flat angled ideal triangulations, we allow some ideal tetrahedra to be flat. This means that the ideal tetrahedron is as shown in Figure 1. More specifically, two nonadjacent edges have interior angle $\pi$, and the remainder have interior angle zero. If two faces of a flat ideal tetrahedron share an edge with interior angle $\pi$, we term them coherent. Thus, the four faces are partitioned into two coherent pairs.

The full definition of a partially flat angled ideal triangulation is given below.

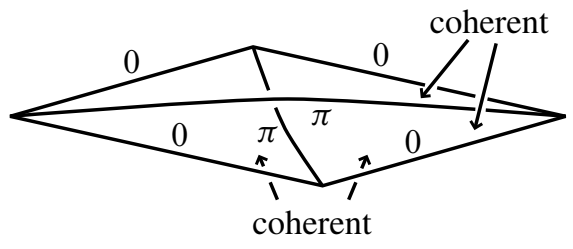

Figure 1: A flat ideal tetrahedron

A layered polygon is a collection of flat ideal tetrahedra glued together in a certain way to form a 3-manifold. It is determined by the following data: an ideal polygon with an initial ideal triangulation, together with a finite sequence of elementary moves applied to this triangulation, subject to the condition that every edge of the initial triangulation that is not in the boundary of the ideal polygon has a move performed upon it at some stage. Recall that an elementary move on an ideal triangulation of a surface removes an edge adjacent to two distinct ideal triangles, forming an ideal square, and then inserts the other diagonal of this square as a new edge. (See Figure 2.)

Starting with this data, we build the layered polygon. We start with the initial ideal triangulation of the ideal polygon, which will be the base of the layered polygon. The first move acts upon a pair of adjacent faces. Attach onto them a flat ideal tetrahedron along a coherent pair of faces. The "top" of the resulting object inherits the second ideal triangulation of the ideal polygon. Repeat this for each move of the sequence. The resulting 3-manifold is the layered polygon. (See Figure 3.) It is a 3-ball with a 


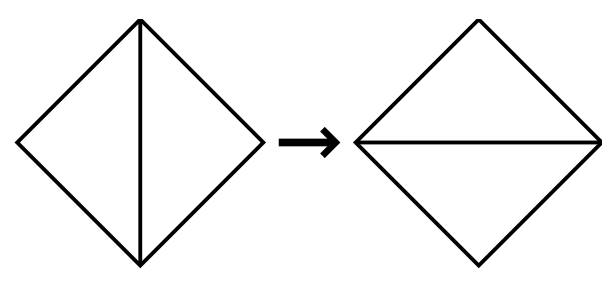

Figure 2: An elementary move

finite collection of points in its boundary removed. Its boundary is the union of two ideal polygons, which are the initial and terminal ideal polygons in the sequence of elementary moves. The intersection of these is a collection of edges, which we term its vertical boundary.

Elementary moves
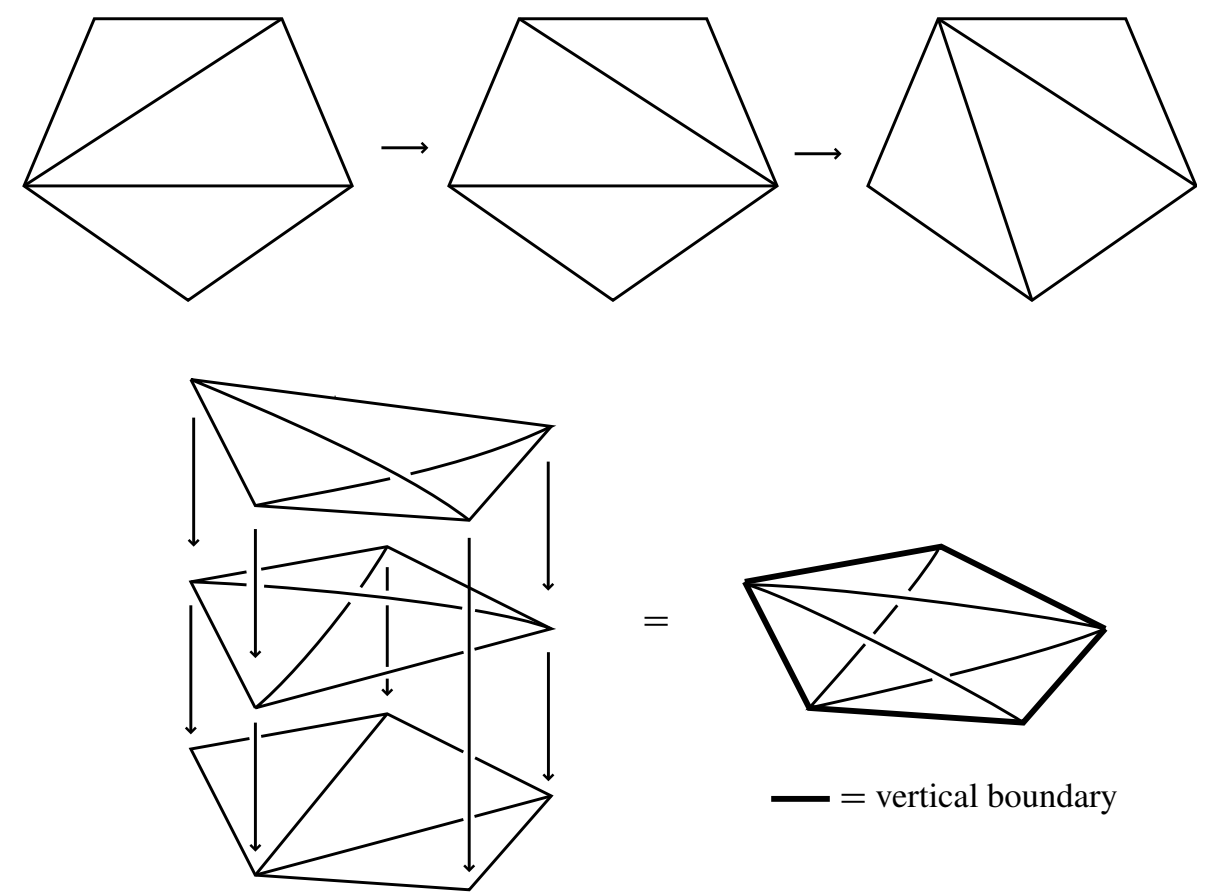

The resulting layered polygon

Figure 3: Construction of a layered polygon 
A partially flat angled ideal triangulation of a 3-manifold is an ideal triangulation, with a real number in the range $[0, \pi]$ assigned to each edge of each ideal tetrahedron, known as the interior angle of the edge, satisfying the following conditions:

(i) the angles at each ideal vertex of each ideal tetrahedron sum to at most $\pi$;

(ii) the angles around each edge sum to $2 \pi$;

(iii) if the angles of an ideal tetrahedron are not all strictly positive, then the ideal tetrahedron is flat;

(iv) the union of the flat ideal tetrahedra is a collection of layered polygons, possibly with some edges in their vertical boundary identified.

Note that, in (i), the angles at each ideal vertex are not required to sum to precisely $\pi$, unlike the case of an angled ideal triangulation. This is so that we can deal with 3-manifolds having some boundary components with negative Euler characteristic.

Note also that we do not allow layered polygons to intersect each other or themselves along anything other than vertical boundary edges. They are not allow to touch at any point in the interior of the "top" or "base" of a layered polygon. (See Figure 4.)

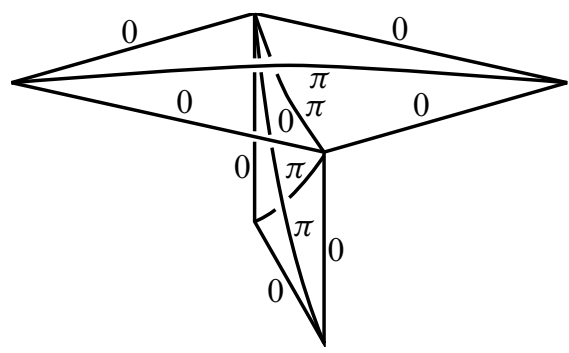

Figure 4: A forbidden arrangement

The usefulness of partially flat angled ideal triangulations is that the normal and almost normal surfaces with nonnegative Euler characteristic that they contain are very constrained. We briefly recall the relevant terminology.

A normal disc in a tetrahedron or ideal tetrahedron is a properly embedded disc that misses the vertices, that hits each edge transversely in at most one point and that is not disjoint from the edges. There are two types of normal discs, triangles and squares, which are shown in Figure 5. A closed surface properly embedded in $M$ is normal if it intersects each ideal tetrahedron in a collection of disjoint normal discs. 


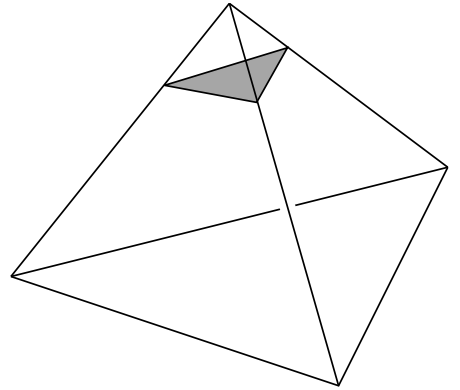

Triangle

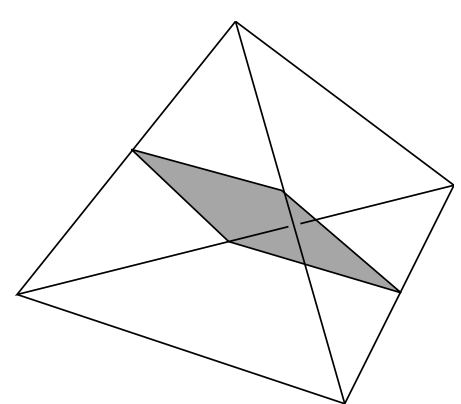

Square

Figure 5: Normal discs

An almost normal piece in a tetrahedron or ideal tetrahedron is one of two types: either an octagon, as shown in Figure 6, or a tubed piece, which is two disjoint normal discs tubed together via a tube that runs parallel to an edge. A closed surface properly embedded in $M$ is almost normal if it intersects each ideal tetrahedron in a collection of normal discs, except in precisely one ideal tetrahedron, where it is a collection of normal discs and exactly one almost normal piece.

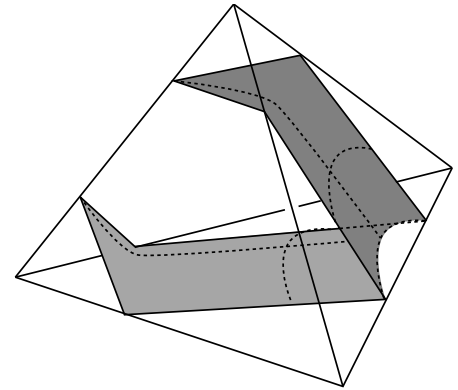

Octagon

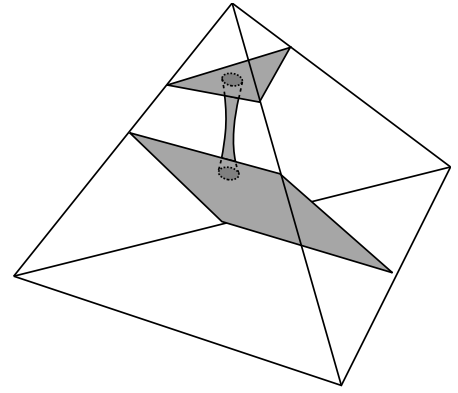

Tubed piece

Figure 6: Almost normal pieces

It is a theorem of Rubinstein [12] and Stocking [16] that any strongly irreducible Heegaard surface in a compact orientable 3-manifold may be ambient isotoped into almost normal form with respect to any given triangulation or ideal triangulation. A variant of this result (Theorem 4.2) will be vital in this paper.

We now examine how normal and almost normal surfaces interact with a partially flat angle structure. We follow Matveev (see Section 5.2 in Matveev [9]) and term a surface 
2-normal if it is closed and embedded and it intersects each ideal tetrahedron in a collection of normal discs and octagons. It will be useful to consider 2-normal surfaces with nonnegative Euler characteristic. Here, we have the following result.

Theorem 2.1 Let $\mathcal{T}$ be a partially flat angled ideal triangulation of a compact orientable 3-manifold $M$. Then any connected closed 2-normal surface in $\mathcal{T}$ with nonnegative Euler characteristic is normally parallel to a toral boundary component of $M$.

The key tool in the proof of this is a quantity known as combinatorial area, which is assigned to any 2-normal (or more general) surface in $M$. The partially flat angle structure assigns an interior angle in the range $[0, \pi]$ to each edge of each ideal tetrahedron. The corresponding exterior angle is defined to be $\pi$ minus the interior angle. The combinatorial area of any normal or almost normal piece is defined to be the sum of the exterior angles of the edges it runs over (counted with multiplicity) minus $2 \pi$ times its Euler characteristic. It is easy to verify that this is always at least zero. Any triangle running over edges with interior angles that sum to $\pi$ has zero area. The only other normal or almost normal piece with zero area is a so-called vertical square in a flat ideal tetrahedron. This is a square that intersects the edges with angle $\pi$. (See Figure 7.)

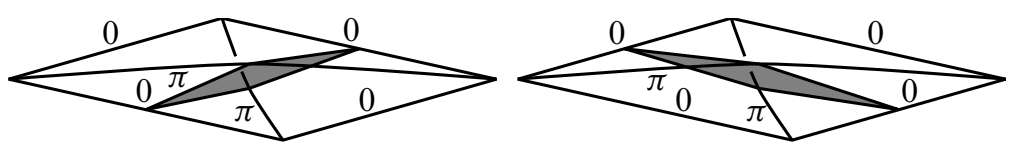

Figure 7: Vertical squares

The combinatorial area of a 2-normal surface $F$ is the sum of the combinatorial areas of its normal and almost normal discs. It is proved in Proposition 4.3 of [6] that this is equal to $-2 \pi \chi(F)$. Thus, $\chi(F)$ is always nonpositive. Suppose, as in the hypothesis of Theorem 2.1, that $\chi(F)$ is also nonnegative. Then, $F$ must be composed entirely of triangles and vertical squares. We claim that in fact $F$ consists only of triangles. Hence, if $F$ is connected, it is normally parallel to a toral boundary component of $M$, as required.

Vertical squares lie inside flat ideal tetrahedra, each of which lies in some layered polygon $P$. Focus on the top ideal tetrahedron of this layered polygon. Its intersection with the top ideal polygon of $P$ is a square $S$. One of the diagonals of this square is an edge in the top ideal polygon. Since no other layered polygons are attached to this edge, 
the normal discs of $F-P$ adjacent to it are all triangles. Hence, every arc of $S \cap F$ separates off a single vertex of $S$. Thus, in the intersection of the top ideal tetrahedron with $F$, there are no vertical squares, only triangles. Repeating this argument for each of the ideal tetrahedra of $P$, we deduce that $F \cap P$ is only triangles, as required.

It is not known whether every finite-volume hyperbolic 3-manifold admits an angled ideal triangulation. But, according to the following existence theorem, it does always have a partially flat angled ideal triangulation.

Theorem 2.2 Let $M$ be a compact connected orientable 3-manifold with nonempty boundary. Let $T$ be its toral boundary components. Then the following are equivalent:

(1) $M$ is simple and not a 3-ball;

(2) $M-T$ admits a finite-volume hyperbolic structure with totally geodesic boundary;

(3) $M$ admits a partially flat angled ideal triangulation.

Moreover, if these conditions are satisfied, there is an algorithm that constructs a partially flat angled ideal triangulation, starting with any triangulation of $M$.

Proof $(1) \Rightarrow(2)$ This is a well known result of Thurston. The proof goes as follows. Let $D M$ be the result of doubling $M$ along $\partial M-T$, and let $D T$ be the two copies of $T$ in $D M$. Then $D M$ is a compact orientable simple Haken 3-manifold with (possibly empty) toral boundary. So, by Thurston's geometrisation theorem [10], $D M-D T$ admits a complete finite-volume hyperbolic structure. There is an involution of $D M$ that interchanges its two halves. By Mostow's rigidity theorem, this is homotopic to an isometry. By a result of Tollefson [17], the involution and the isometry are equivariantly isotopic. The fixed-point set of this isometry is therefore a totally geodesic copy of $\partial M-T$ in $D M$. This divides $D M-D T$ into two copies of $M-T$, each of which inherits a finite-volume hyperbolic structure with totally geodesic boundary, as required.

(2) $\Rightarrow$ (3) It is a theorem of Epstein and Penner [2] that, when $\partial M=T$, the interior of $M$ is obtained from a finite collection of hyperbolic ideal polyhedra, by gluing their faces isometrically in pairs. When $\partial M$ strictly contains $T$, there is a version of this theorem, due to Kojima [5]. Instead of hyperbolic ideal polyhedra, one uses truncated hyperbolic hyperideal polyhedra. Recall that these are defined as follows. Use the projective model for hyperbolic space $\mathbb{H}^{3}$, which is the open ball in projective 3-space. A hyperideal polyhedron is the intersection of this open ball with a polyhedron $P$, such that every vertex of $P$ lies outside of $\mathbb{H}^{3}$, but where no edge of $P$ lies completely outside of $\mathbb{H}^{3}$. Thus, some vertices of $P$ lie on the sphere at 
infinity of $\mathbb{H}^{3}$ (these are the ideal vertices), and some lie outside the sphere at infinity (these are the hyperideal vertices). Each hyperideal vertex of $P$ is at the apex of a cone tangent to the sphere at infinity of $\mathbb{H}^{3}$. The intersection of this cone with the sphere at infinity is a circle, which bounds a totally geodesic plane in $\mathbb{H}^{3}$. If one truncates the polyhedron along each of these planes and removes any vertices on the sphere at infinity, the result is a truncated hyperbolic hyperideal polyhedron. The faces of a truncated hyperbolic hyperideal polyhedron are totally geodesic and come in two types: interior faces, which are subsets of faces of the original polyhedron $P$, and exterior faces, which are subsets of the truncating hyperplanes. We permit all the vertices of $P$ to lie on the sphere at infinity, and so a hyperbolic ideal polyhedron is a special case of a hyperbolic hyperideal polyhedron and a special case of a truncated hyperbolic hyperideal polyhedron. Kojima's theorem states that $M-T$ can be obtained from a finite collection of truncated hyperbolic hyperideal polyhedra by gluing their interior faces isometrically in pairs. The exterior faces patch together to form $\partial M-T$.

Pick a vertex of each polyhedron $P$ as above, that arises in the decomposition of $M-T$ into truncated hyperbolic hyperideal polyhedra. We call this the coning vertex of $P$. The polyhedron $P$ is therefore a cone on this vertex, the base of the cone being those faces that do not contain the vertex. If we subdivide each of these faces into triangles, then coning these off at the coning vertex induces a decomposition of the hyperideal polyhedron into hyperideal tetrahedra. (See Figure 8.) Each hyperideal tetrahedron inherits a set of nonzero interior angles, satisfying condition (i) in the definition of a partially flat angled ideal triangulation.

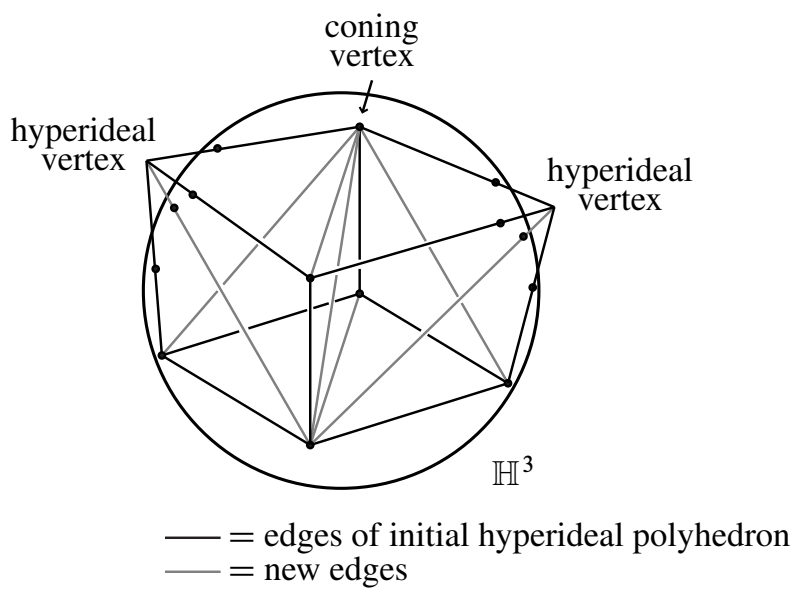

Figure 8 
This decomposition of the hyperideal polyhedron yields a decomposition of each its faces into topological ideal triangles. These topological ideal triangles may not be hyperbolic ideal triangles because some of their vertices may lie outside the sphere at infinity. When two faces of the decomposition are glued isometrically, their topological ideal triangulations may not agree. However, these two ideal triangulations differ by a finite sequence of elementary moves, which we may assume leaves no edge in the interior of the faces untouched. Insert the corresponding layered polygon between the two faces, interpolating between their ideal triangulations. (See Figure 9.) Thus, we obtain an ideal triangulation of the 3-manifold with an angle assignment to each edge of each ideal tetrahedron. It is clear that the conditions (i), (ii), (iii) and (iv) in the definition of a partially flat angled ideal triangulation are satisfied.

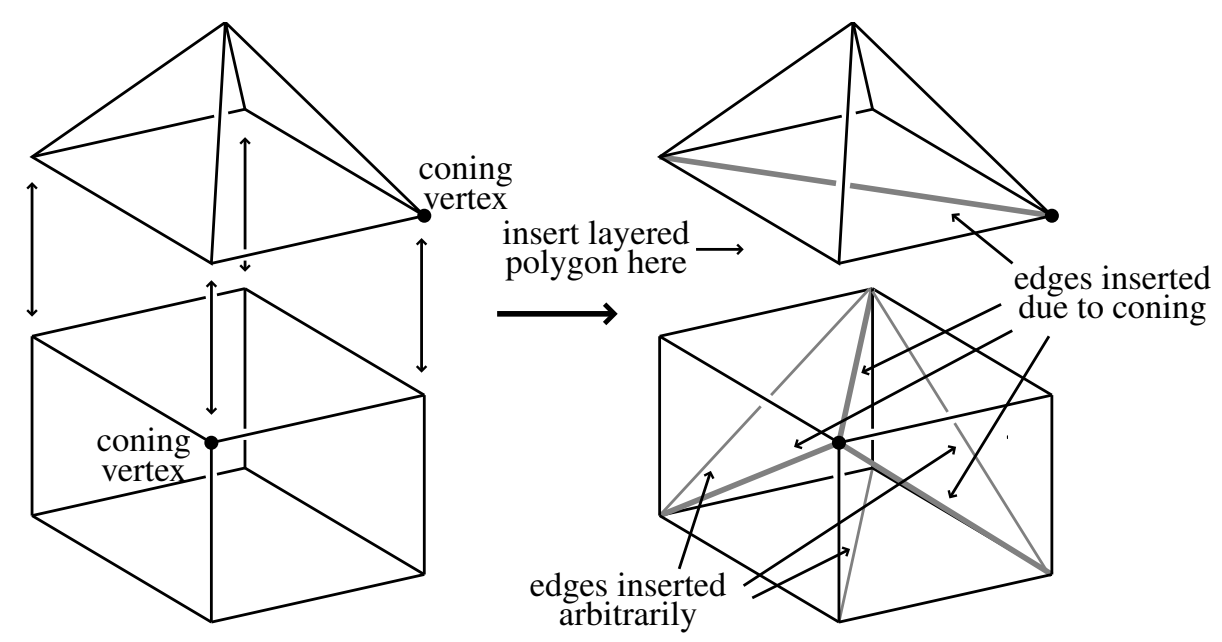

Figure 9

(3) $\Rightarrow$ (1) This is essentially contained in Corollary 4.6 in [6]. We sketch the proof now. Suppose that $M$ admits a partially flat angled ideal triangulation. If $M$ is reducible, then it contains a normal 2-sphere, contrary to Theorem 2.1. If $M$ contains a properly embedded incompressible torus, then this can be ambient isotoped into normal form, and hence is boundary parallel by Theorem 2.1. In order to deal with properly embedded discs and incompressible annuli in $M$, we need to introduce a definition of normal surfaces that intersect $\partial M$ and to prove a version of Theorem 2.1 for these. We will not give the full details here, but refer the reader instead to Proposition 4.5 in [6]. Thus, $M$ is simple. Also, $M$ cannot be a 3-ball, for one could then find a normal 2-sphere parallel to $\partial M$, contradicting Theorem 2.1. 
Let us now suppose that $M$ has a partially flat angled ideal triangulation. We need to give an algorithm to find one. Starting with a triangulation of the manifold, there is a simple algorithm that constructs an ideal triangulation (see Theorem 1.1.13 of Matveev [9]). Given any ideal triangulation, there is an algorithm that determines whether it admits a partially flat angle structure, since this is just a linear programming problem. Any two ideal triangulations of a compact orientable 3-manifold differ by a sequence of 2-3 and 3-2 moves (see Figure 10), by a result of Matveev (Theorem 1.2.5 of [9]).
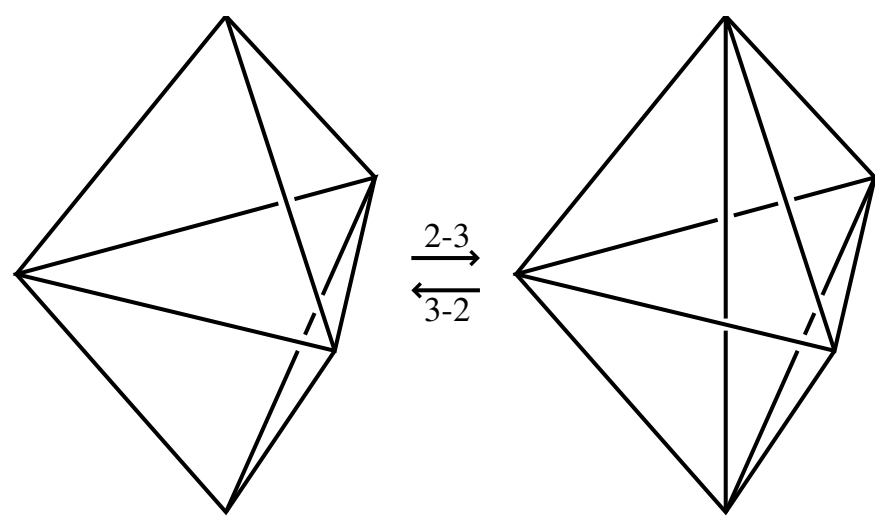

Figure 10: 2-3 and 3-2 moves

Thus, the algorithm to construct the partially flat angled ideal triangulation proceeds as follows. One checks whether the initial ideal triangulation admits a partially flat angle structure. If it does, we are done and we stop. If not, then one applies all possible 2-3 and 3-2 moves to the ideal triangulation, giving a new collection of ideal triangulations. One checks each of these for partially flat angle structures. Continuing in this fashion, a partially flat angled ideal triangulation is eventually constructed.

\section{Generalised Heegaard splittings}

It is technically convenient, when dealing with Heegaard surfaces, to focus on those that are strongly irreducible. The piece of machinery that allows one to make this reduction is known as untelescoping, which yields a generalised Heegaard splitting for the manifold. We now briefly describe these concepts.

Recall that a compression body $C$ is a connected orientable 3-manifold that either is a handlebody or is obtained from $S \times[0,1]$ by attaching 1 -handles to $S \times\{1\}$, where $S$ 
is a closed orientable, possibly disconnected, surface. The copy of $S \times\{0\}$ in $C$ is termed the negative boundary and is denoted $\partial_{-} C$. The negative boundary is defined to be empty when $C$ is a handlebody. The remainder of $\partial C$ is the positive boundary and is denoted $\partial_{+} C$. A handle structure on $C$ is an expression of $C$ as either $\partial_{-} C \times I$ with 1-handles attached, or as a 3-ball with 1-handles attached. Note that, in general, a compression body has many different handle structures.

A generalised Heegaard splitting of a compact orientable 3-manifold $M$ is a decomposition of the manifold along closed orientable disjoint properly embedded separating surfaces into manifolds $C_{1}, \ldots, C_{m}$, each of which is a disjoint union of compression bodies, such that $\partial_{-} C_{2 i} \cap \operatorname{int}(M)=\partial_{-} C_{2 i+1} \cap \operatorname{int}(M)$ and $\partial_{+} C_{2 i}=\partial_{+} C_{2 i-1}$ for each relevant integer $i$. Let $F_{i}$ be the surface $C_{i} \cap C_{i+1}$. This is known as an even or odd surface depending on the parity of $i$. We view the even surfaces as dividing $M$ into a collection of 3-manifolds, and the odd surfaces as forming Heegaard splittings for these manifolds. (See Figure 11.)

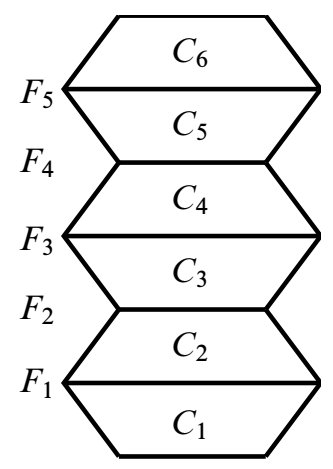

Figure 11: A generalised Heegaard splitting

There is a method for constructing a Heegaard splitting for a 3-manifold, starting with a generalised Heegaard splitting $\left\{C_{1}, \ldots, C_{m}\right\}$, known as amalgamation (see Schultens [15]). This procedure is a sequence of modifications, each of which we term a partial amalgamation. Each partial amalgamation is based around one of the even surfaces, $F_{2}$, say. Either side of this even surface, there are two collections of compression bodies $C_{2}$ and $C_{3}$. Pick a handle structure on each of these compression bodies that is not a handlebody. Thus, we view each such compression body as obtained from $F_{2}^{\prime} \times I$, where $F_{2}^{\prime} \times\{0\}$ is the relevant components of $F_{2}$, by attaching a collection of 1-handles to $F_{2}^{\prime} \times\{1\}$. We extend each of these 1-handles vertically through $F_{2}^{\prime} \times I$, so that they are attached to $F_{2}$. We may ensure that the attaching discs of these 1handles are all disjoint. Let $F_{1}^{\prime}$ be the surface obtained from $F_{2}$ by attaching these 
tubes. It separates $C_{1} \cup C_{2} \cup C_{3} \cup C_{4}$ into two collections of compression bodies $C_{1}^{\prime}$ and $C_{2}^{\prime}$, where $C_{1}^{\prime}$ is a copy of $C_{1}$ with 1 -handles attached, and $C_{2}^{\prime}$ is a copy of $C_{4}$ with 1-handles attached. We therefore end with a new generalised Heegaard splitting $\left\{C_{1}^{\prime}, C_{2}^{\prime}, C_{5}, \ldots, C_{m}\right\}$ for $M$, which is obtained from the previous one by partial amalgamation. (See Figure 12.) When this procedure is performed as many times as possible, the result is a Heegaard splitting, which is an amalgamation of the original generalised Heegaard splitting.

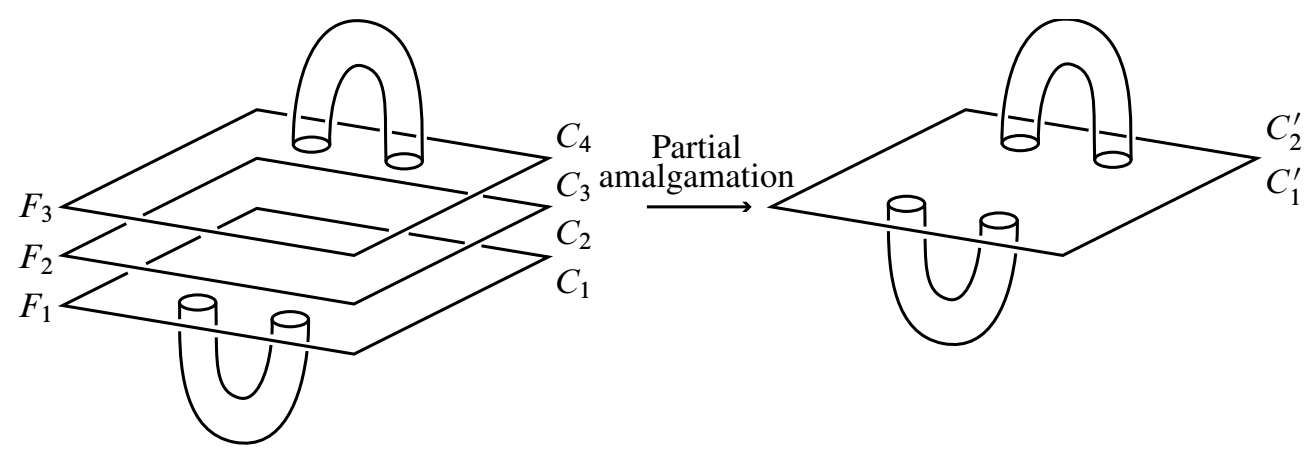

Figure 12

Choices were made when forming the Heegaard splitting for $M$ : we picked handle structures on $C_{2}$ and $C_{3}$, and we picked an order on the even surfaces in which to perform the partial amalgamations. It is in fact the case that the resulting Heegaard splitting of $M$ is independent of these choices. This important result does not appear to be present in the literature, and so we provide a proof.

Proposition 3.1 If one amalgamates a generalised Heegaard splitting, the resulting Heegaard splitting is well-defined up to ambient isotopy. In particular, it is independent of the order of partial amalgamations and the choice of handle structures on the compression bodies.

Let us first examine what happens when we change the order of the partial amalgamations. Each partial amalgamation is based around an even surface. So, consider two such even surfaces, and the associated partial amalgamations. We must show that if one swaps the order of these partial amalgamations, the resulting generalised Heegaard splitting is unchanged up to ambient isotopy. This is clear if the indexing integers of the even surfaces differ by more than 2 , because in this case none of the compression bodies involved in the different partial amalgamations intersect. Thus, we focus on the case where the indexing integers of the even surfaces differ by 2: say that they 
are $F_{2}$ and $F_{4}$. Now, we may view the former partial amalgamation procedure as the removal of $F_{1}$ and $F_{2}$, and the addition of handles onto $F_{3}$. Similarly, the latter partial amalgamation can be viewed as the removal of $F_{4}$ and $F_{5}$, together with addition of handles onto $F_{3}$. So, whatever the order of the two partial amalgamations, the resulting odd surface is the same: it is $F_{3}$ with handles attached to both sides.

Let us now consider what happens when we vary the handle structure on one of the compression bodies $C$ that is not a handlebody. This handle structure is determined by the co-cores of the 1-handles, which form a collection $D$ of disjoint compression discs for $\partial_{+} C$. This collection is complete, in the sense that the result of compressing $\partial_{+} C$ along $D$ is a copy of $\partial_{-} C$. There is clearly a one-one correspondence between handle structures on $C$ (up to ambient isotopy) and complete collections of compression discs for $\partial_{+} C$ (up to ambient isotopy). Thus, we are led to the question of how two different complete collections of compression discs are related. The following answer is well known (see Proposition B.1 of Bonahon [1] for example).

Lemma 3.2 Any two complete collections of compression discs for a compression body differ by a finite sequence of band moves.

The definition of a band move is as follows. Let $D_{1}$ and $D_{2}$ be distinct discs in a complete collection $D$. Let $\alpha$ be an arc in $\partial_{+} C$ with interior disjoint from $D$ and with one endpoint in $D_{1}$ and the other endpoint in $D_{2}$. Let $N$ be a regular neighbourhood of $D_{1} \cup \alpha \cup D_{2}$. Then, $\operatorname{cl}\left(\partial N-\partial_{+} C\right)$ consists of three compression discs for $\partial_{+} C$, one parallel to $D_{1}$, one parallel to $D_{2}$, and a third which we denote by $D_{1}^{\prime}$. Then, $D \cup D_{1}^{\prime}-D_{1}$ is a new complete collection of compression discs, obtained from $D$ by a band move.

When the compression body $C$ is embedded within a 3 -manifold $M$, as in the current situation, we can realise these band moves by handle slides as follows. We view $C$ as $\partial_{-} C \times[0,1]$, with $1-$ handles attached to $\partial_{-} C \times\{1\}$. Let $D=\left\{D_{1}, D_{2}, \ldots, D_{n}\right\}$ be the co-cores of the 1-handles, and let $D^{\prime}=\left\{D_{1}^{\prime}, D_{2}, \ldots, D_{n}\right\}$ be obtained from $D$ by a band move along $\alpha$, as above. Now isotope the $1-$ handle corresponding to $D_{2}$, by sliding its attaching disc incident to $\alpha$ along $\alpha$, and then over $D_{1}$. The new compression body $C^{\prime}$ is clearly ambient isotopic to $C$, but now $D^{\prime}$ is ambient isotopic in $C^{\prime}$ to the cocores of its 1-handles. See Figure 13.

Let us now consider two different ways of performing a partial amalgamation upon a generalised Heegaard splitting $\left\{C_{1}, \ldots, C_{m}\right\}$. Let us suppose, for the sake of being definite, that these partial amalgamations are centred on the surface $\partial_{-} C_{2}=\partial_{-} C_{3}$. Let us also suppose that these two partial amalgamations are specified by the same handle structures on $C_{2}$, but where the handle structures on $C_{3}$ differ by a handle 


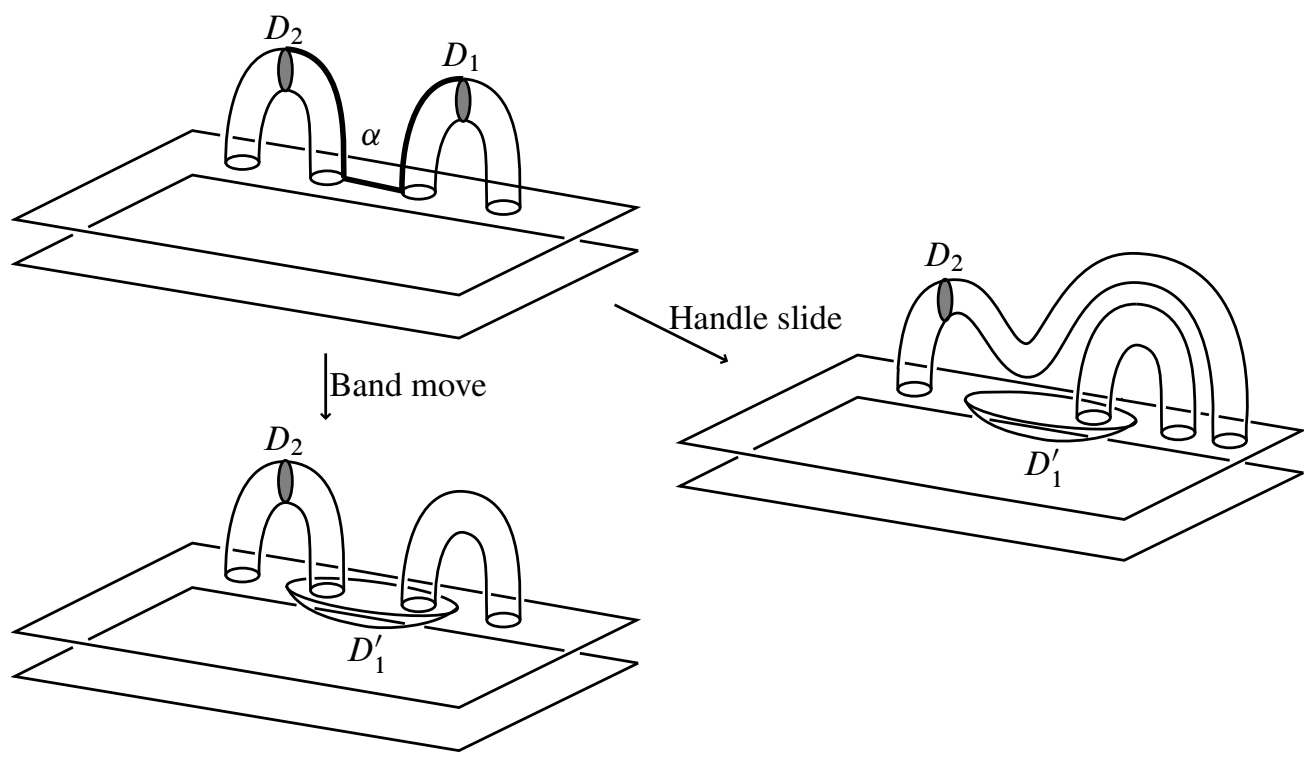

Figure 13

slide. We view $C_{3}$ as $\partial_{-} C_{3} \times[0,1]$, with 1 -handles attached in two different ways. The second set of handles is obtained from the first set, by sliding one of the attaching discs of one of the handles along an arc $\alpha^{\prime}$ in $\partial_{-} C_{3} \times\{1\}$ up to another handle and over that handle. When we perform the first partial amalgamation, the new odd surface $F_{1}^{\prime}$ is obtained from $\partial_{-} C_{3}$ by attaching handles onto both sides. By using the product structure on $\partial_{-} C_{3} \times[0,1]$, we may project the arc $\alpha^{\prime}$ to an arc in $\partial_{-} C_{3}$. By slightly isotoping this arc if necessary, we may assume that its interior avoids the attaching discs of all the handles. This arc joins two handles of $F_{1}^{\prime}$. We may therefore slide one of these handles along this arc, and over the other handle. The resulting surface $F_{1}^{\prime \prime}$ is exactly that obtained by the second partial amalgamation. Thus, $F_{1}^{\prime}$ and $F_{1}^{\prime \prime}$ are related by a handle slide and are therefore ambient isotopic. (See Figure 14.)

We have therefore shown that the choices made in creating the amalgamated Heegaard surface do not affect its ambient isotopy class. This proves Proposition 3.1.

We will be constructing the Heegaard splittings required by Theorem 1.1 by first constructing generalised Heegaard splittings. Thus, we need to know that the process of amalgamation can be achieved algorithmically. 

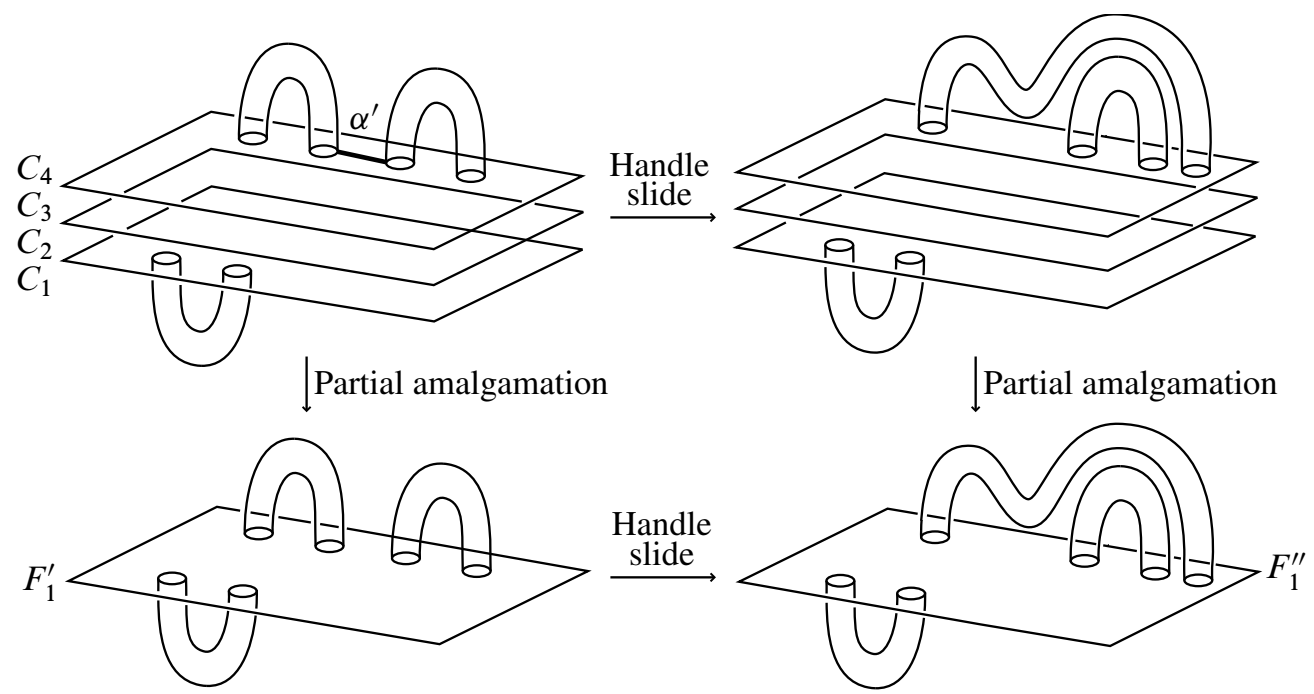

Figure 14

Proposition 3.3 Let $F$ be a Heegaard surface for $M$ that is obtained from a generalised Heegaard splitting $\left\{C_{1}, \ldots, C_{m}\right\}$ by amalgamation. Suppose that $\left\{C_{1}, \ldots, C_{m}\right\}$ is given as a subcomplex of a triangulation of $M$. Then, there is an algorithm that constructs $F$ in $M$.

Proof Let $\left\{C_{1}^{\prime}, C_{2}^{\prime}, C_{5}, \ldots, C_{m}\right\}$ be obtained from $\left\{C_{1}, \ldots, C_{m}\right\}$ by a partial amalgamation. It clearly suffices to construct $C_{1}^{\prime}$ and $C_{2}^{\prime}$ from $C_{1}, C_{2}, C_{3}$ and $C_{4}$. Let us focus on a component of $C_{3}$, say, that is not a handlebody. A complete set of compression discs for this compression body is constructible (see Theorem 4.1.14 of Matveev [9] or Algorithm 9.3 of Jaco and Tollefson [4]). Cutting along this collection, we obtain a copy of $F_{2}^{\prime} \times[0,1]$, where $F_{2}^{\prime}$ is the relevant components of $\partial_{-} C_{3}$. In $F_{2}^{\prime} \times\{1\}$, we have two copies of each compression disc, giving a collection $D^{\prime}$ of disjoint discs. We may construct $D^{\prime} \times[0,1]$ in $F_{2}^{\prime} \times[0,1]$ as follows. In $F_{2}^{\prime} \times[0,1]$, we may construct a vertical annulus $A$, using Theorem 6.4.10 of [9] or Algorithm 9.7 of [4]. By performing an ambient isotopy on $A$ supported in a small neighbourhood of $F_{2}^{\prime} \times\{1\}$, we may ensure that $A$ intersects each component of $D^{\prime}$ in a nonempty collection of arcs. For each disc $D^{\prime \prime}$ in $D^{\prime}$, we may construct a properly embedded arc in $A$ running from a component of $D^{\prime \prime} \cap A$ to $F_{2}^{\prime} \times\{0\}$. We may arrange that these arcs are pairwise disjoint. Let $R$ be their union. Then, a regular neighbourhood of $R \cup D^{\prime}$ in $F_{2}^{\prime} \times[0,1]$ is the required copy of $D^{\prime} \times[0,1]$. If we now reverse the cutting procedure which gave $F_{2}^{\prime} \times[0,1]$ from the component of $C_{3}$, the components 
of $D^{\prime} \times[0,1]$ glue up in pairs to form a collection of 1 -handles attached to $\partial_{-} C_{3}$. Perform this procedure for each compression body component of $C_{2}$ and $C_{3}$, and then isotope if necessary, so that the attaching discs of the 1-handles in $\partial_{-} C_{2}=\partial_{-} C_{3}$ are disjoint. The new surface $\partial_{-} C_{1}^{\prime}=\partial_{-} C_{2}^{\prime}$ is obtained from $\partial_{-} C_{2}$ by attaching these tubes.

The following result was proved by Scharlemann and Thompson [14]. It describes a process known as untelescoping.

Theorem 3.4 Let $M$ be a compact orientable 3-manifold, and let $F$ be an irreducible Heegaard surface. Then there is a generalised Heegaard splitting $\left\{C_{1}, \ldots, C_{m}\right\}$ for $M$, such that

(i) the even surfaces are incompressible and have no 2-sphere components;

(ii) the odd surfaces are strongly irreducible;

(iii) no $C_{i}$ is homeomorphic to $\partial_{-} C_{i} \times I$ (although some components of $C_{i}$ may be products);

(iv) $F$ is obtained from this generalised Heegaard splitting by amalgamation.

Suppose, in addition, that the Heegaard genus of $M$ is more than 1. Then, we may also arrange that no odd surface is composed entirely of tori.

We now wish to estimate the genus of the odd and even surfaces in this generalised Heegaard splitting. Let us suppose that the Heegaard genus of $M$ is more than 1 . Now, it is trivial to check that the quantity

$$
\sum_{i=1}^{m} \frac{\chi\left(\partial_{-} C_{i}\right)-\chi\left(\partial_{+} C_{i}\right)}{2}
$$

is unchanged under partial amalgamation (see Lemma 2 in Scharlemann and Schultens [13]). Hence, it equals $-\chi(F)+(\chi(\partial M) / 2)$. Each term in the sum is a positive integer, by (iii) and the fact that no $C_{i}$ is a collection of solid tori, and no component of any $C_{i}$ is a $3-$ ball. Thus, we obtain the inequalities $m \leq-\chi(F)+(\chi(\partial M) / 2) \leq-\chi(F)$. Since $F$ is obtained from the splitting by amalgamation, it can be viewed as obtained from any given even or odd surface by adding tubes. Thus, the genus of each even or odd surface is at most $g(F)$, the genus of $F$. The number of even and odd surfaces is $m-1 \leq 2 g(F)-3$. So, the genus of the union of the odd and even surfaces is at most $g(F)(2 g(F)-3)$. (It is possible to improve this estimate slightly, but all that is needed here is a computable upper bound on the genus of the union of the odd and even surfaces in terms of $g(F)$.) 


\section{Almost normal surfaces}

This paper relies heavily on the following important theorem of Rubinstein [12] and Stocking [16].

Theorem 4.1 Let $M$ be a compact orientable irreducible 3-manifold, with a given triangulation. Let $F$ be a strongly irreducible Heegaard surface for $M$. Then there is an ambient isotopy taking $F$ into almost normal form.

In this paper, we need the following slight extension of this result, which deals also with ideal triangulations and with generalised Heegaard splittings.

Theorem 4.2 Let $M$ be a compact orientable irreducible 3-manifold, with a given triangulation or ideal triangulation. Let $\left\{C_{1}, \ldots, C_{m}\right\}$ be a generalised Heegaard splitting for $M$. Suppose that the even surfaces are incompressible and have no $2-$ sphere components and the odd surfaces are strongly irreducible. Then there is an ambient isotopy that makes each even surface normal and each component of the odd surfaces almost normal.

The proof follows the argument of Stocking in [16] almost word-for-word. We refer the reader to [16] for more details.

For our purposes here, the main usefulness of normal and almost normal surfaces is that they are constructible.

Theorem 4.3 Let $\mathcal{T}$ be a partially flat angled ideal triangulation of a compact orientable 3-manifold $M$. Then, for any integer $n, \mathcal{T}$ contains only finitely many closed orientable properly embedded surfaces $F$ with genus $(F) \leq n$, and where each component of $F$ is either normal or almost normal. Moreover, there is an algorithm to construct each of these surfaces.

The remainder of this section is devoted to proving this result.

Let $F$ be a closed orientable properly embedded surface, each component of which is normal or almost normal. Let $\bar{F}$ be obtained from $F$ by compressing any tubed pieces. Thus, $\bar{F}$ is 2 -normal, and genus $(\bar{F}) \leq \operatorname{genus}(F) \leq n$. So, it clearly suffices to construct a finite list of possibilities for $\bar{F}$. For we may then reconstruct $F$ by reattaching tubes running parallel to the edges of $\mathcal{T}$. Note that, according to Theorem 2.1, $\mathcal{T}$ contains no 2-normal 2-spheres. Hence, each of the compressions we performed on $F$ was essential. 
Now, $\bar{F}$ may be specified by a vector, each coordinate of which is a nonnegative integer, as follows. One associates with each ideal tetrahedron 10 coordinates. Each coordinate corresponds to a type of normal or almost normal disc in that tetrahedron: 4 triangle types, 3 square types and 3 octagon types. The vector corresponding to $\bar{F}$ simply counts the number of copies in $\bar{F}$ of each normal and almost normal disc in each ideal tetrahedron. The fact that these discs patch together to form a closed surface forces this vector to satisfy certain linear equations. There are three equations for each face of the ideal triangulation, corresponding to the three types of properly embedded arc in that face. These are known as the matching equations. An embedded surface cannot contain different square or octagon types in any given ideal tetrahedron. This again forces constraints on the vector of $\bar{F}$. For normal surfaces, these are known as the quadrilateral conditions. In our situation, we will term them the square/octagon conditions. There is a one-one correspondence between closed properly embedded 2 -normal surfaces and nonnegative integer solutions to the matching equations that satisfy the square/octagon conditions. (See Remark 1 on p 204 in [9]).

Crucial is the concept of normal sum. Suppose that the vector corresponding to $\bar{F}$ can be written as a sum of vectors, each of which has nonnegative integer coordinates and satisfies the matching equations. Then these vectors also satisfy the square/octagon conditions and so correspond to 2 -normal surfaces $F_{1}$ and $F_{2}$. We write $\bar{F}=F_{1}+F_{2}$. It is easy to check that $\chi(\bar{F})=\chi\left(F_{1}\right)+\chi\left(F_{2}\right)$. When $\bar{F}$ cannot be written as a sum of nonempty 2 -normal surfaces, $\bar{F}$ is said to be fundamental. Crucial to our algorithms is the following fact (see Theorem 3.2.8 of [9]).

Lemma 4.4 There is a finite computable list of fundamental surfaces, such that any 2-normal surface may be written as a sum of these fundamental surfaces.

Denote these fundamental surfaces by $F_{1}, \ldots, F_{m}$. Suppose that $F_{1}, \ldots, F_{r}$ are normally parallel to toral boundary components of $M$ and that the rest are not. Thus, Lemma 4.4 states that any 2 -normal surface $\bar{F}$ can be written as $\sum_{i=1}^{m} n_{i} F_{i}$, for nonnegative integers $n_{i}$. Consider $\sum_{i=r+1}^{m} n_{i} F_{i}$, which is a solution to the matching equations satisfying the square/octagon conditions. It therefore corresponds to a $2-$ normal surface $F^{\prime}$. According to Theorem $2.1, \chi\left(F_{i}\right)$ is strictly negative for each $i>r$. Hence, it is at most -1 , and we obtain the inequalities

$$
\sum_{i=r+1}^{m} n_{i} \leq-\sum_{i=r+1}^{m} n_{i} \chi\left(F_{i}\right)=-\sum_{i=1}^{m} n_{i} \chi\left(F_{i}\right)=-\chi(\bar{F})=2 g(\bar{F})-2|\bar{F}|<2 g(\bar{F}) \leq 2 n .
$$

Thus, there is a finite list of possibilities for $F^{\prime}$ and they are all constructible. 
The surface $\sum_{i=1}^{r} n_{i} F_{i}$ is a collection of copies of the toral boundary components, which we may realise as disjoint from $F^{\prime}$. Thus, the union of these surfaces and $F^{\prime}$ is a solution to the matching equations with the same vector as $\bar{F}$. They are therefore ambient isotopic. In other words,

Hence

$$
\begin{aligned}
\bar{F} & =F^{\prime} \cup \bigsqcup_{i=1}^{r} n_{i} F_{i} . \\
g(\bar{F}) & =g\left(F^{\prime}\right)+\sum_{i=1}^{r} n_{i} .
\end{aligned}
$$

Since we are assuming that the genus of $\bar{F}$ is at most $n$, this provides an upper bound on $\sum_{i=1}^{r} n_{i}$. Thus there is a finite list of possibilities for $\bar{F}$ and they are all constructible. The same is then true for $F$. This completes the proof of Theorem 4.3.

\section{The algorithms}

We now have all the ingredients to describe the algorithms in Theorem 1.1 and to prove that they work. Note that the first algorithm, which computes the Heegaard genus of $M$, can be constructed from the second algorithm, which finds all Heegaard surfaces with genus at most a given integer $n$. This is done as follows. One first sets $n$ to be 2 (the smallest possible Heegaard genus for $M$ ) and one searches for Heegaard surfaces with genus at most $n$. If there is one, the Heegaard genus is 2 . If there is not, set $n$ to be 3 , and repeat. The first time the algorithm finds a Heegaard surface, it necessarily has minimal genus, and the algorithm stops.

Therefore, let us fix a nonnegative integer $n$. We will describe the algorithm to find all Heegaard surfaces in $M$ with genus at most $n$.

We may restrict attention to irreducible Heegaard surfaces. For if a Heegaard surface in $M$ is reducible, it is stabilised, and is therefore obtained from an irreducible Heegaard surface of smaller genus by stabilising a number of times.

Step 1 Find a partially flat angled ideal triangulation for $M$.

The algorithm to achieve this is described in the proof of Theorem 2.2. The algorithm finds not just the required ideal triangulation with an explicit partially flat angle structure, but also provides a method of constructing it from the initial given triangulation. 
Step 2 Find candidates for generalised Heegaard splittings.

According to Theorem 3.4, given any irreducible Heegaard surface $F$ for $M$, there is a generalised Heegaard splitting, from which $F$ is obtained by amalgamation, and in which each even surface is incompressible and has no 2-sphere components, and each odd surface is strongly irreducible. Let $F^{\prime}$ be the union of the even and odd surfaces. As observed at the end of Section 3, the conclusions of Theorem 3.4 imply that the genus of $F^{\prime}$ is at most $g(F)(2 g(F)-3)$. By Theorem 4.2, we may make each even surface normal and each component of the odd surfaces almost normal. According to Theorem 4.3, there is an algorithm that constructs a finite list of surfaces in $M$, one of which is $F^{\prime}$. Step 2 in the algorithm is to construct this list of surfaces.

Step 3 Determine which are generalised Heegaard splittings.

There is an algorithm to determine whether a properly embedded closed, possibly disconnected, surface $F^{\prime}$ forms a generalised Heegaard splitting. It proceeds as follows. Cut $M$ along $F^{\prime}$. There is an algorithm that determines whether each component of the complement is a compression body (see Theorem 4.1.14 of [9] or Algorithm 9.3 of [4]). If this holds, the algorithm then checks all possible ways of grouping these compression bodies into an ordered collection $\left\{C_{1}, \ldots, C_{m}\right\}$ (where each $C_{i}$ may be disconnected) such that $\partial_{-} C_{2 i} \cap \operatorname{int}(M)=\partial_{-} C_{2 i+1} \cap \operatorname{int}(M)$ and $\partial_{+} C_{2 i}=\partial_{+} C_{2 i-1}$ for each relevant integer $i$. We apply this algorithm to each surface provided by Step 2, and thereby create a list of generalised Heegaard splittings.

Step 4 Amalgamation.

Consider one of the generalised Heegaard splittings $\left\{C_{1}, \ldots, C_{m}\right\}$ in our list, and suppose that $F$ is the Heegaard surface obtained from this by amalgamation. According to Proposition 3.1, this surface $F$ depends only on the generalised Heegaard splitting, and not on any choices made during the amalgamation procedure. If one is interested only in the genus of $F$, then this can be calculated from the surfaces in the generalised Heegaard splitting via the formula

$$
-\chi(F)+\frac{\chi(\partial M)}{2}=\sum_{i=1}^{m} \frac{\chi\left(\partial_{-} C_{i}\right)-\chi\left(\partial_{+} C_{i}\right)}{2} .
$$

Thus, if one is interested only in the existence of a Heegaard surface with genus at most $n$, then this can be determined by applying this formula to each generalised Heegaard splitting in the list. The algorithm discards all those Heegaard surfaces with genus more than $n$. However, if one actually wants to construct all such Heegaard surfaces, then one must perform each amalgamation algorithmically, using Proposition 3.3. The result is a finite list of Heegaard surfaces for $M$ with genus at most $n$. 
We close this paper with a proof of Corollary 1.2. Let $L$ be a hyperbolic link in the 3 -sphere. We now describe an algorithm that supplies, for any given positive integer $n$, all tunnel systems for $L$ with at most $n$ arcs, up to slide-equivalence. Let $M$ be the exterior of $L$, which satisfies the hypotheses of Theorem 1.1. There is a one-one correspondence between tunnel systems for $L$ (up to slide-equivalence) and Heegaard surfaces for $M$ (up to ambient isotopy) that bound a handlebody on one side. If the tunnel system has $n$ arcs, the Heegaard surface has genus $n+1$. The algorithm of Theorem 1.1 constructs all Heegaard surfaces for $M$ with genus at most $n+1$, up to ambient isotopy. It is straightforward to discard those that do not bound a handlebody on one side. However, to complete the proof of Corollary 1.2, we must give a method of constructing a tunnel system from a Heegaard surface $F$ that bounds a handlebody on one side. On the other side of $F$ is a compression body $C$. A complete set of compression discs for $\partial_{+} C$ in $C$ is constructible (see Theorem 4.1.14 of [9] or Algorithm 9.3 of [4]). Cutting $C$ along this collection gives a copy of $\partial_{-} C \times[0,1]$, where $\partial_{-} C \times\{0\}=\partial_{-} C$. In $\partial_{-} C \times\{1\}$, we have two copies of each compression disc. Let $D^{\prime}$ be the union of these discs. As in the proof of Proposition 3.3, we may construct a collection of properly embedded vertical arcs $R$ in $\partial_{-} C \times[0,1]$ such that their intersection with $\partial_{-} C \times\{1\}$ lies in $D^{\prime}$ and intersects each component of $D^{\prime}$ in a single point. By performing an ambient isotopy supported in a small neighbourhood of $\partial_{-} C \times\{1\}$, we may arrange that these arcs glue up in pairs when the discs of $D^{\prime}$ are re-identified. Thus, they form a tunnel system for $L$.

\section{References}

[1] F Bonahon, Cobordism of automorphisms of surfaces, Ann. Sci. École Norm. Sup. (4) 16 (1983) 237-270 MR732345

[2] D B A Epstein, R C Penner, Euclidean decompositions of noncompact hyperbolic manifolds, J. Differential Geom. 27 (1988) 67-80 MR918457

[3] W Jaco, J Rubinstein, Layered-triangulations of 3-manifolds arXiv: math/0603601

[4] W Jaco, J L Tollefson, Algorithms for the complete decomposition of a closed 3manifold, Illinois J. Math. 39 (1995) 358-406 MR1339832

[5] S Kojima, Polyhedral decomposition of hyperbolic 3-manifolds with totally geodesic boundary, from: "Aspects of low-dimensional manifolds", Adv. Stud. Pure Math. 20, Kinokuniya, Tokyo (1992) 93-112 MR1208308

[6] M Lackenby, Word hyperbolic Dehn surgery, Invent. Math. 140 (2000) 243-282 MR1756996

Algebraic $\&$ Geometric Topology, Volume 8 (2008) 
[7] T Li, Heegaard surfaces and measured laminations. II. Non-Haken 3-manifolds, J. Amer. Math. Soc. 19 (2006) 625-657 MR2220101

[8] T Li, Heegaard surfaces and measured laminations. I. The Waldhausen conjecture, Invent. Math. 167 (2007) 135-177 MR2264807

[9] S Matveev, Algorithmic topology and classification of 3-manifolds, Algorithms and Computation in Math. 9, Springer, Berlin (2003) MR1997069

[10] J W Morgan, On Thurston's uniformization theorem for three-dimensional manifolds, from: "The Smith conjecture (New York, 1979)", Pure Appl. Math. 112, Academic Press, Orlando, FL (1984) 37-125 MR758464

[11] C Petronio, J R Weeks, Partially flat ideal triangulations of cusped hyperbolic 3manifolds, Osaka J. Math. 37 (2000) 453-466 MR1772844

[12] J H Rubinstein, Polyhedral minimal surfaces, Heegaard splittings and decision problems for 3-dimensional manifolds, from: "Geometric topology (Athens, GA, 1993)", AMS/IP Stud. Adv. Math. 2, Amer. Math. Soc. (1997) 1-20 MR1470718

[13] M Scharlemann, J Schultens, The tunnel number of the sum of $n$ knots is at least $n$, Topology 38 (1999) 265-270 MR1660345

[14] M Scharlemann, A Thompson, Thin position for 3-manifolds, from: "Geometric topology (Haifa, 1992)", Contemp. Math. 164, Amer. Math. Soc. (1994) 231-238 MR1282766

[15] J Schultens, The classification of Heegaard splittings for (compact orientable surface) $\times S^{1}$, Proc. London Math. Soc. (3) 67 (1993) 425-448 MR1226608

[16] M Stocking, Almost normal surfaces in 3-manifolds, Trans. Amer. Math. Soc. 352 (2000) 171-207 MR1491877

[17] J L Tollefson, Involutions of sufficiently large 3-manifolds, Topology 20 (1981) 323352 MR617370

Mathematical Institute, University of Oxford

24-29 St Giles', Oxford OX1 3LB, United Kingdom

lackenby@maths.ox.ac.uk

Received: 7 February 2008 Revised: 1 May 2008 